\title{
MOBILIDADE ARTICULAR DOS DEDOS NÃO LESADOS PÓS-REPARO EM LESÃO DOS TENDÕES FLEXORES DA MÃO
}

\author{
Rabelo RB ${ }^{1}$, Fonseca MCR ${ }^{2}$, Mazzer $\mathrm{N}^{2}$, Elui VMC ${ }^{3}$ e Barbieri $\mathrm{CH}^{2}$ \\ ${ }^{1}$ Fisioterapeuta \\ ${ }^{2}$ Departamento de Biomecânica, Medicina e Reabilitação do Aparelho Locomotor, Universidade de São Paulo - USP, \\ Ribeirão Preto, SP - Brasil \\ ${ }^{3}$ Departamento de Terapia Ocupacional, USP \\ Correspondência para: Marisa de Cássia Registro Fonseca, Departamento de Biomecânica, Medicina e Reabilitação do \\ Aparelho Locomotor, Universidade de São Paulo, Av. Bandeirantes, 3900, CEP 14049-900, Ribeirão Preto, SP - Brasil, \\ e-mail: marisa@fmrp.usp.br e rafa_rehabil@yahoo.com.br
}

Recebido: 08/11/2006 - Revisado: 04/04/2007 - Aceito: 28/06/2007

\begin{abstract}
RESUMO
Objetivo: Verificar a amplitude de movimento (ADM) em mãos que sofreram reparo tendinoso dos músculos flexores superficial e profundo dos dedos, comparando os dados de cada dedo na mão lesada e entre mãos lesadas e não lesadas. Métodos: Foi realizada a goniometria ativa em 15 pacientes e 120 dedos, 60 dedos de mãos lesadas e 60 de mãos controle não lesadas. Os sujeitos foram avaliados no momento da retirada da tala gessada, tendo sido realizada a movimentação precoce pelo método de Duran modificado. A partir dos dados goniométricos, foram registrados os valores do índice TAM (Total Active Motion) dos dedos nas mãos lesadas e controle. Para análise dos dados, foi acessada a fórmula de índices funcionais proposta pela American Society for Surgery of the Hand (ASSH) e para cálculo estatístico, foi escolhido o Modelo de Efeitos Mistos. Resultados: A fórmula da ASSH para os dedos lesados mostrou que 18,33\% tiveram a classificação do movimento "bom”, 18,33\%, "regular" e 63,34\%, “pobre”. Foram comparadas as médias das medidas em graus de todos os dedos entre si dentro de cada grupo, controle ou lesado, e as médias das medidas entre os grupos, encontrando-se um p-valor significante apenas entre os grupos controle e lesado. Não houve diferença estatística entre o TAM de cada dedo na mão lesada. Conclusão: Independente de quantos dedos tenham sofrido lesão tendinosa em uma mão, os dedos não lesados também terão suas ADMs ativas diminuídas no período logo após a retirada da imobilização.
\end{abstract}

Palavras-chave: amplitude de movimento; lesão dos tendões flexores; quadriga.

\section{ABSTRACT \\ Joint range of motion of uninjured fingers after repairs to flexor tendon injuries of the hand}

Objective: To assess the range of motion (ROM) in hands that underwent tendon repair in the flexor digitorum superficialis and flexor digitorum profundus muscles of the fingers, comparing the data between the fingers on the injured hand, and between the injured and uninjured hands. Method: Active goniometry was performed on 15 patients, making a total of 120 fingers (60 on injured hands and 60 on noninjured control hands). The patients were examined at the time of removing the plaster splint. Early mobilization was performed using the modified Duran method. Goniometric data were used for recording the TAM (total active motion) values of the fingers on the injured and uninjured (control) hands. To analyze the data, the functional index formula proposed by the American Society for Surgery of the Hand (ASSH) was utilized, and for statistical calculations the mixed-effect model was selected. Results: The ASSH formula for the injured fingers classified the movement as "good" in 18.33\%, "fair" in $18.33 \%$ and "poor" in $63.34 \%$. The means, in degrees, of the measurements for all the fingers were compared with each other within each group (control and injured) and between the groups. A significant difference was found between control and injured groups. There was no statistical difference between the TAM of each finger on the injured hand. Conclusion: Independent of how many fingers on one hand had suffered tendon injuries, the uninjured fingers also presented diminished active ROM during the period immediately after removal of the immobilization.

Key words: range of motion; flexor tendon injuries; quadriga. 


\section{INTRODUÇÃO}

O efeito de diminuição da amplitude de movimento em dedos não lesados após ruptura tendinosa é uma complicação comum observada na prática clínica. Esse impedimento da excursão tendinosa dos dedos não envolvidos na lesão primária foi primeiramente descrito por Bunnell ${ }^{1}$ em 1924.

Bunnell ${ }^{1}$ notou o fenômeno após amputação de um dedo, descrevendo uma técnica na qual os tendões do flexor profundo e extensor foram suturados distalmente junto ao osso. O autor explica que, por fazerem parte de um ventre muscular comum, a restrição da mobilidade em um tendão limita a excursão dos tendões restantes.

Mais tarde, o mesmo fenômeno foi chamado de quadriga por Verdan², que compara o ventre e os tendões do flexor profundo dos dedos a uma carroça com suas rédeas, como a carroça romana do "Circus Maximus".

A síndrome da quadriga ocorre como conseqüência de um bloqueio, geralmente resultante de aderência, que impede o deslizamento tendinoso do flexor profundo dos dedos (FPD) $)^{3,4}$. Ocorre decréscimo da excursão dos tendões adjacentes aos lesados, causando decréscimo da força e amplitude de movimento nesses dedos ${ }^{3}$.

Outros trabalhos demonstram que o fenômeno não está apenas ligado ao bloqueio do FPD, mas também está relacionado a estruturas anatômicas que interconectam músculos ou tendões como a bainha sinovial comum dos flexores dos dedos no túnel do carpo ${ }^{5}$, a existência de tendões anômalos conectando o músculo flexor longo do polegar ao flexor profundo do $2^{\circ}$ e $3^{\circ}$ dedos ${ }^{6}$, as interconexões que ligam a aponeurose do extensor comum dos dedos às metacarpofalangianas (MFs) ${ }^{7}$, aderências tendinosas em lesões do músculo flexor superficial dos dedos ${ }^{8}$, anomalias do tendão flexor em pacientes com síndrome do túnel do carpo $^{9}$. Esses estudos sugerem que o deslizamento tendinoso ou muscular que estiver envolvido em uma determinada ação deve ser harmônico, e que fibras ligamentares, tendões anômalos, retináculos, aderências ou qualquer estrutura anatômica que conecte um tendão ou músculo ao outro tornam o movimento estruturalmente interdependente.

Embora o efeito quadriga ocorra comumente na prática clínica, sua relação com a diminuição da ADM e força é pouco demonstrada em pesquisas.

O objetivo deste estudo é analisar a amplitude de movimento (ADM) dos dedos adjacentes aos que sofreram reparo tendinoso dos músculos flexores superficial e/ou profundo em uma mesma mão. Este propósito é baseado na hipótese de que os tendões flexores dos dedos têm função muscular interdependente e, por isso, estão sujeitos ao efeito quadriga.

Também é proposta deste trabalho mostrar que as leituras goniométricas dos dedos da mão lesada estão agrupadas e são interdependentes, tendo correspondência estatística, permitindo a comparação de mãos lesadas independente do número de dedos atingidos ou da zona em que o tendão sofreu ferimento.

\section{MATERIAIS E MÉTODOS}

\section{Cuidados éticos}

O estudo e coleta de dados deste trabalho foram aprovados pelo Comitê de Ética em Pesquisa do Hospital das Clínicas da Faculdade de Medicina de Ribeirão Preto da Universidade de São Paulo (HCFMRP-USP), de acordo com o Processo HCRP n ${ }^{\circ} .12270 / 2004$. Os sujeitos recrutados assinaram um termo de consentimento livre e esclarecido.

\section{Sujeitos}

Foram coletados dados de 15 pacientes $(n=15)$, sendo 8 homens e 7 mulheres, com idade média de 24,27 anos (13 a 46 anos), com lesão tendinosa unilateral.

Os pacientes recrutados, obrigatoriamente, foram operados na Unidade de Emergência do HCFMRP-USP para serem submetidos à movimentação passiva precoce pelo protocolo de Duran modificado ${ }^{10}$, sendo acompanhados pelo Serviço de Fisioterapia do referido hospital.

O ferimento poderia ser associado ou não a lesão nervosa e vascular e a lesões dos flexores do punho.

Foram excluídos os casos de reimplantes, lesões complexas associadas a fraturas, queimaduras ou perda de substância da pele da mão, além de politraumatizados, lesões neurológicas centrais prévias e lesões bilaterais.

Em 10 pacientes, a área referente à lesão foi a zona topográfica flexora $\mathrm{V}$, correspondente ao punho; em 4 pacientes, a lesão ocorreu na zona II, região do túnel osteofibroso volar dos dedos; e em 1 paciente, a lesão ocorreu na zona III, na palma da mão. Essas zonas flexoras, correspondentes a 5 áreas topográficas, foram descritas por Verdan e Michon em 1961 e adotadas pela Federação Internacional de Cirurgia da Mão ${ }^{11}$.

Todos os pacientes analisados tiveram lesão nervosa e/ou vascular associada aos ferimentos tendinosos. Dessas lesões, 3 foram do nervo ulnar, 3 com associações dos nervos ulnar e mediano, 3 do nervo mediano e apenas 6 pacientes não tiveram lesão nervosa. Não houve neurorrafia de nervos digitais.

\section{Técnica cirúrgica}

Na tenorrafia primária, o tipo de sutura e a espessura do fio foram os mesmos em todos os pacientes: o tipo de nó da sutura da porção central na união dos cotos foi tipo Kessler com fio de nylon 3-0 e a sutura epitendinosa periférica para chuleio foi realizada com nylon de espessura 5-0.

A sutura tipo Kessler ${ }^{12}$ possibilita a movimentação passiva precoce. A combinação de sutura na porção central que une os cotos com sutura epitendinosa periférica ou chuleio parece ser suficiente para suportar a resistência antecipada que exigem os protocolos de movimentação precoce passiva ou 
ativa $^{13}$. O chuleio, quando associado a outras suturas, aumenta a força do reparo de $20 \%$ a $50 \%$, reduzindo a proporção de rupturas ou falhas no reparo ${ }^{14-16}$.

Não houve casos de ruptura do tendão suturado, infecção pós-operatória ou deiscência na cicatriz cirúrgica. Foram excluídos do estudo pacientes que retiraram a tala gessada antes das três primeiras semanas pós-operatórias.

\section{Procedimentos}

Inicialmente, a partir do primeiro dia pós-operatório, todos os pacientes foram submetidos a um protocolo de movimentação passiva precoce baseado no método de Duran Modificado.

O protocolo de Duran Modificado, proposto por Strickland e Glogovac ${ }^{10}$, é uma modificação do protocolo original sugerido por Duran e Houser ${ }^{17}$. Uma tala dorsal gessada imobiliza o punho e MFs em flexão e mantém as interfalangianas (IF) em extensão neutra. Nesse período a tala não pode ser retirada.

O grau de flexão do punho sofreu variação individual de acordo com o distanciamento dos cotos tendinosos, nervosos ou vasculares no momento do reparo. Quanto mais extensa a lesão tecidual, maior foi o grau de flexão do punho para a união dos cotos. O grau de flexão das MFs foi em torno de $45^{\circ}$.

O protocolo teve duração mínima de 12 semanas a partir do primeiro dia pós-operatório. Nas três primeiras semanas, os exercícios ocorreram na tala gessada e consistiram em movimentação passiva isolada e composta das IF dos dedos em todo arco de movimento, nos limites da órtese. As séries foram de 6 a 8 repetições, três vezes ao dia. A partir da quarta semana, quando foi retirada definitivamente a tala gessada, iniciou-se a movimentação ativa, explorando o efeito tenodese e exercícios de deslizamento tendinoso.

Os exercícios realizados imediatamente após a retirada da tala gessada para os pacientes que ficaram imobilizados com mais de $30^{\circ}$ de flexão de punho foram de movimentação ativa ou ativo-assistida, explorando o arco de movimento conseguido pelo paciente, evitando o tensionamento do reparo.

A goniometria (Figura 1) foi realizada imediatamente após a retirada da órtese na quarta semana pós-operatória. $\mathrm{O}$ instrumento utilizado neste estudo foi um goniômetro digital plástico (North Coast Medical, Inc., San Jose, CA, USA).

As medidas foram coletadas das mãos lesadas e não lesadas, sendo as últimas a representação do grupo controle do estudo. Foi utilizado o sistema de análise goniométrica da ASSH $^{18}$ baseado no índice TAM (Movimento Ativo Total). Esse sistema é largamente utilizado na literatura ${ }^{19}$ e recomendado pela Federação Internacional das Sociedades de Cirurgia da Mão. TAM é a soma da flexão ativa simultânea das articulações MF e IF proximal e distal de um mesmo dedo, subtraindo-se a soma do déficit da extensão total das mesmas articulações ${ }^{20-22}$. A medida é realizada para cada dedo.
No sistema de análise goniométrica da $\mathrm{ASSH}^{19,23}$, o valor TAM é dividido por 260 e multiplicado por 100, totalizando a porcentagem de amplitude ativa dos dedos.

\section{Análise dos dados}

Para análise os dados, foram separados dois grupos: grupo das mãos com dedos lesados, em que foi colhida a goniometria de todos os dedos, independente do número de dedos lesados e grupo controle, no qual foram analisados todos os dedos longos das mãos não lesadas. No total, foi realizada a goniometria de 120 dedos, sendo 60 dedos do grupo controle não lesado e 60 dedos do grupo das mãos lesadas. A comparação dos dados foi realizada intragrupo e intergrupo.

\section{Análise estatística}

Os dados usados neste estudo tiveram sua origem: da comparação dos índices TAM dos dedos lesados em relação aos adjacentes não lesados na mesma mão; da comparação dos índices TAM entre os dedos das mãos não lesadas no grupo controle; da comparação dos índices TAM entre os dois grupos: grupo controle e grupo das mãos com dedos lesados, e dos índices de resultados funcionais, em porcentagem, propostos pela $\mathrm{ASSH}^{19}$.

No sistema de análise funcional da ASSH, os valores são classificados em excelente (100\%) - TAM $\geq 260^{\circ}$; bom (75 a 99\%) - TAM entre $195^{\circ}$ e $259^{\circ}$; regular (50 a $74 \%$ ) TAM entre $130^{\circ}$ e $194^{\circ}$; e pobre $(<50 \%)$ - TAM $<130^{\circ}$.

Foi escolhido como método para cálculo estatístico o Modelo de Efeitos Mistos ${ }^{24}$. O modelo linear de efeitos mistos (efeitos aleatórios e fixos) é utilizado na análise de dados em que as respostas de um mesmo indivíduo estão agrupadas e a suposição de independência entre as observações num mesmo grupo não é adequada ${ }^{24}$.

\section{RESULTADOS}

A Tabela 1 apresenta os resultados dos 60 dedos da mão lesada segundo o sistema de análise funcional da ASSH. A fórmula mostrou que 18,33\% dos dedos lesados tiveram o TAM entre $195^{\circ}$ e $259^{\circ}$, sendo a classificação do movimento "bom"; 18,33\% dos dedos lesados tiveram o TAM entre $130^{\circ}$ e $194^{\circ}$, sendo a classificação do movimento "regular" e $63,34 \%$ dos dedos lesados tiveram o TAM menor que $130^{\circ}$, sendo classificados como movimento "pobre”.

A Tabela 2 compara o TAM dos dedos lesados com o TAM dos dedos controle, representados pela mão contralateral não lesada, expressando o valor em porcentagem. O TAM do dedo contralateral, sem lesão, representa o valor padrão (100\%) para cada paciente. Destaque deve ser dado aos pacientes 6, 9, 13 e 14. Nesses pacientes, observa-se que independente do tendão lesado, profundo e/ou superficial, os dedos não envolvidos na lesão também têm sua mobilidade prejudicada. No paciente 6, que apresentou lesão do nervo 
Tabela 1. Avaliação do TAM dos dedos da mão lesada de acordo com a classificação da American Society for Surgery of the Hand. Valores arbitrários $(\mathrm{n}=15)(60$ dedos$)$.

\begin{tabular}{cccc}
\hline $\begin{array}{c}\text { Classificação } \\
\text { (ASSH) }\end{array}$ & Dedos & TAM & $\begin{array}{c}\text { Porcentagem de movimento } \\
\text { normal (ASSH) }\end{array}$ \\
\hline Excelente & 0 & $\geq 260^{\circ}$ & $100 \%$ \\
Bom & 11 & $195^{\circ}$ a $259^{\circ}$ & $75 \%$ a $99 \%$ \\
Regular & 11 & $130^{\circ}$ a $194^{\circ}$ & $50 \%$ a $74 \%$ \\
Pobre & 38 & $<130^{\circ}$ & $<50 \%$ \\
\hline
\end{tabular}

TAM= Total Active Motion, ASSH= American Society for Surgery of the Hand.

Tabela 2. Valores do índice TAM (Total Active Motion) dos dedos da mão lesada em relação ao TAM do dedo controle. Valores arbitrários $(\mathrm{n}=15)$ (120 dedos).

\begin{tabular}{|c|c|c|c|c|c|c|}
\hline \multicolumn{7}{|c|}{ TAM dedo lesado / TAM dedo controle $\mathrm{x}_{\mathrm{x} 100}$} \\
\hline & & & $2^{\circ}$ dedo & $3^{\circ}$ dedo & $4^{\circ}$ dedo & $5^{\circ}$ dedo \\
\hline Paciente 1 & $\mathbf{V}$ & $\oplus$ & $44,23 \%$ & $23,40 \%$ & $22,12 \%^{\bullet *}$ & $16,00 \%^{\bullet *}$ \\
\hline Paciente 2 & $\mathbf{V}$ & $\otimes$ & $10,86 \%$ & $15,10 \%{ }^{\bullet *}$ & $13,04 \%^{\bullet *}$ & $6,12 \%^{\bullet *}$ \\
\hline Paciente 3 & $\mathbf{V}$ & $\varnothing$ & $23,80 \%$ & $36,17 \%$ & $32,00 \%$ & $32,00 \%$ \\
\hline Paciente 4 & $\mathbf{V}$ & $\varnothing$ & $39,00 \%$ & $58,50 \%$ & $63,82 \%$ & $83,67 \%$ \\
\hline Paciente 5 & $\mathbf{V}$ & $\otimes$ & $7,70 \%$ & $5,70 \%$ & $7,40 \%$ & $3,63 \%^{\bullet}$ \\
\hline Paciente 6 & $\mathbf{V}$ & $\oplus$ & $52,00 \%$ & $16,00 \%$ & $16,67 \%$ & $9,44 \%$ \\
\hline Paciente 7 & II & $\Theta$ & $48,00 \%$ & $91,00 \%$ & $83,00 \%$ & $94,00 \%$ \\
\hline Paciente 8 & II & $\Theta$ & $45,28 \%{ }^{\bullet *}$ & $34,54 \%{ }^{\bullet *}$ & $23,63 \%^{\bullet *}$ & $27,00 \%{ }^{\bullet *}$ \\
\hline Paciente 9 & III & $\Theta$ & $25,00 \%{ }^{\bullet *}$ & $62,50 \%$ & $74,00 \%$ & $67,40 \%$ \\
\hline Paciente 10 & $\mathbf{V}$ & $\oplus$ & $87,50 \%$ & $81,63 \%$ & $51,00 \%$ & $38,46 \%$ \\
\hline Paciente 11 & $\mathbf{V}$ & $\otimes$ & $23,00 \%{ }^{\bullet *}$ & $15,68 \%^{\bullet *}$ & $15,10 \%^{\bullet *}$ & $12,30 \%^{\bullet *}$ \\
\hline Paciente 12 & $\mathrm{~V}$ & $\varnothing$ & $7,84 \%^{\bullet *}$ & $12,00 \%^{\bullet *}$ & $8,34 \%^{\bullet *}$ & $10,42 \%$ \\
\hline Paciente 13 & II & $\Theta$ & $72,00 \%$ & $70,00 \%$ & $86,00 \%$ & $49,00 \%{ }^{*}$ \\
\hline Paciente 14 & II & $\Theta$ & $25,00 \%{ }^{*}$ & $62,00 \%$ & $58,00 \%$ & $58,00 \%$ \\
\hline Paciente 15 & $\mathrm{~V}$ & $\Theta$ & $98,00 \%$ & $98,00 \%$ & $96,00 \%$ & $96,00 \%{ }^{*}$ \\
\hline
\end{tabular}

Porcentagens em negrito representam os dedos lesados. • Lesão flexor superficial dos dedos, * Lesão flexor profundo dos dedos, $\oplus$ lesão nervo ulnar, $\varnothing$ lesão nervo mediano, $\otimes$ lesão associada dos nervos mediano e ulnar, $\Theta$ ausência de lesão nervosa. Áreas de lesão: II (zona II), III (zona III), V (zona V).

ulnar e lesão dos tendões flexor superficial do $3^{\circ}$ e $4^{\circ}$ dedos, os dedos adjacentes laterais não envolvidos na lesão também tiveram prejuízo na movimentação ativa total.

$\mathrm{Na}$ análise estatística, que analisou a interação intra e intergrupo, foi utilizado o Modelo de Efeitos Mistos ${ }^{24}$.

Os valores da média, desvio-padrão e valores mínimo e máximo do TAM, nos grupos controle e lesado, são apresentadas na Tabela 3. Essa tabela mostra que as médias do grupo controle foram significativamente maiores que a do grupo lesado, havendo diferença significativa intergrupo. A comparação entre as médias dos valores TAM para os dedos, dentro de cada grupo, não indicou diferença estatisticamente significativa, não havendo, portanto, diferença intragrupo. 
Tabela 3. Média, desvio-padrão e valores máximo e mínimo do TAM (Total Active Motion) dos dedos do grupo controle e lesado. Valores arbitrários (graus) ( $\mathrm{n}=15)$ (120 dedos).

\begin{tabular}{ccccc}
\hline Grupo & Dedo & Média & Mínimo & Máximo \\
\hline \multirow{6}{*}{ Controle } & $2^{\circ}$ & $243,33 \pm 15,07$ & 210,00 & 265,00 \\
& $3^{\circ}$ & $257,66 \pm 12,79$ & 235,00 & 280,00 \\
& $5^{\circ}$ & $252,00 \pm 15,21$ & 230,00 & 275,00 \\
& $2^{\circ}$ & $99,33 \pm 69,86$ & 20,00 & 255,00 \\
Lesado & $3^{\circ}$ & $116,46 \pm 79,82$ & 15,00 & 255,00 \\
& $4^{\circ}$ & $109,13 \pm 79,76$ & 20,00 & 250,00 \\
& $5^{\circ}$ & $101,33 \pm 83,01$ & 10,00 & 255,00 \\
\hline
\end{tabular}

\section{DISCUSSÃO}

A fórmula para análise goniométrica da ASSH é comumente utilizada na avaliação dos resultados pós-reparo dos tendões flexores dos dedos ${ }^{18}$. Nessa fórmula, as leituras goniométricas são apresentadas dedo a dedo, como uma série de observações isoladas, o que dificulta a interpretação dos dados para análise funcional da mão.

Os resultados apresentados por este estudo demonstram que mãos com diferentes números de dedos lesados podem ser comparadas, sendo as medidas de ADM interdependentes para cada grupo. Apenas um dedo lesado pode caracterizar uma mão com todas as suas medidas articulares alteradas. Portanto, as leituras goniométricas não devem ser analisadas como medidas isoladas. A mão deve ser vista como uma estrutura com partes harmônicas e interdependentes e não como um conjunto de dedos com mobilidade isolada e aleatória.

A diminuição da mobilidade articular dos dedos não envolvidos na lesão tendinosa primária ocorreu independente de a lesão ter sido isolada ou em associação com os flexores profundo e/ou superficial e de o ferimento ter sido ou não acompanhado de lesão nervosa. Esse fenômeno pode ser justificado pelo efeito quadriga ${ }^{2}$, em que ferimentos tendinosos dos flexores dos dedos retratam prejuízos da função do músculo como um todo e, portanto, de todos os dedos envolvidos na ação muscular.

A compreensão da anatomia do músculo FPD ajuda a entender o efeito quadriga. No terço médio do antebraço, o ventre muscular do FPD separa-se em duas porções, uma radial e outra ulnar. No terço distal, a porção radial forma 0 tendão profundo do dedo indicador, permitindo a flexão isolada da interfalangiana distal (IFD) sem a flexão simultânea das IFDs dos outros três dedos. A porção ulnar forma o tendão profundo para os três dedos mediais. Esses tendões possuem conexões tendinosas que não permitem a flexão independente da IFD do $3^{\circ}$, $4^{\circ}$, e $5^{\circ}$ dedos $25-27$.

Além disso, os tendões dos flexores profundos do $3^{\circ}$, $4^{\circ}$ e $5^{\circ}$ dedos têm conexões através do terceiro e quarto lumbricais. Os dois lumbricais mediais são bipenados e têm origem nos lados adjacentes dos três tendões mediais do flexor profundo ${ }^{28}$.

Estudos demonstram que o efeito quadriga pode ocorrer devido a ligamentos, links musculares ou até mesmo aderências $^{5-9}$ que estejam conectando um tendão ou músculo ao outro, o que pode acometer não apenas o FPD, mas qualquer outro músculo envolvido.

Acredita-se que, após ferimentos cortantes, ocorra perda proprioceptiva, justificada pela lesão nos mecanoceptores das estruturas envolvidas. Os mecanoceptores responsáveis pela informação proprioceptiva são primariamente encontrados nos músculos, tendões, ligamentos e cápsula articular ${ }^{29-34}$. Essa teoria reforça, ainda mais, a relação interdependente entre os dedos da mão e o efeito quadriga.

Concordando com este estudo, Baktir et al. ${ }^{35}$ não encontraram diferença na mobilidade articular entre mãos com mais de um dedo lesado ou aquelas com apenas um dedo lesado. Em seus achados, lesão apenas do tendão flexor profundo ou superficial dos dedos ou ambos os tendões não afetaram os resultados.

Conclui-se que a lesão do tendão flexor de apenas um dedo prejudicará a função de todos os dedos da mão após a cirurgia de tenorrafia, estando os flexores profundo e superficial dos dedos sujeitos ao efeito quadriga. Na análise da avaliação goniométrica, a comparação pode ser realizada entre mãos que sofreram lesão e a contralateral não lesada, ou entre duas mãos lesadas, independente do número de dedos lesados.

Acredita-se que este trabalho traz resultados e propostas importantes para a pesquisa que visa à aplicação prática e teórica. Enfatiza-se que há necessidade de novos estudos científicos na área de cirurgia e terapia da mão pela dificuldade que enfrentam o cirurgião e o terapeuta especializados no tratamento imediato e na reabilitação desses pacientes.

Apoio financeiro: Fundação de Amparo à Pesquisa do Estado de São Paulo - FAPESP; processo $n^{\circ}$ 04/15200-6.

\section{REFERÊNCIAS BIBLIOGRÁFICAS}

1. Bunnell S. Reconstructive surgery of the hand. Surg Gynecol Obstetr. 1924;34:259-74.

2. Verdan C. Syndrome of the quadriga. Surg Clin North Am. 1960;40:425-6.

3. Neu BR, Murray JF, MacKenzie JK. Profundus tendon blockage: quadriga in finger amputations. J Hand Surg. 1985;10:878-83.

4. Lister G. Pitfalls and complications of flexor tendon surgery. Hand Clin. 1985;1:133-46. 
5. Fahrer M. Reparative surgery of flexor tendons in the digits. In: Verdan C, Boyes JH. Tendon surgery of the hand. $1^{\text {a }}$ ed. New York: Churchill Livingstone; 1979. p. 17-24.

6. Linburg RM, Comstock BE. Anomalous tendon slips from the flexor pollicis longus to the flexor digitorum profundus. J Hand Surg. 1979;4:79-83.

7. Wilhelm A. The quadriga phenomenon of the extensor tendon system and the superficial transverse metacarpal ligament. Handchir Mikrochir Plast Chir. 1988;20(4):173-9.

8. Guignard RM. Incomplete section of a superficial flexor tendon in zone II: successive complications and quadriga syndrome. Ann Chir Main Memb Super. 1991;10(4):354-9.

9. Slater RR. Flexor tendon anomalies in a patient with carpal tunnel syndrome. J Hand Surg. 2001;26B:373-6.

10. Strickland JW, Glogovac SV. Digital function following flexor tendon repair in zone II: a comparison study of immobilization and controlled passive motion. J Hand Surg. 1980;5A:537-43.

11. Verdan C, Michon J. Le traitement des plaies des tendons fléchisseuers des doigts. Rev Chir Orthop. 1961;47:285-425.

12. Kessler I. The "grasping" technique for tendon repair. Hand. 1973;5:253-5.

13. Rosenthal EA, Stoddard CW. Questions hand therapists ask about treatment of tendon injuries. J Hand Ther. 2005;18: 313-8

14. Silfverskiöld KL, May EJ, Tornvall AH. Flexor digitorum profundus tendon excurtions during controlled motion after flexor tendon repair in zone II: a prospective clinical study. J Hand Surg. 1992;17A:122.

15. Strickland JW, Cannon N. Post flexor tendon repair motion protocol. Indiana Hand Center Newsletter. 1993;1(1):13-8.

16. Strickland JW. Development of flexor tendon surgery: twentyfive years of progress. J Hand Surg. 2000;25A:214-35.

17. Duran RJ, Hauser RG. Controlled passive motion following flexor tendon repair in zone II and III. In: Hunter JM, Schneider LH, editores. AAOS symposium on tendon surgery in the hand. St Louis: CV Mosby; 1975. p. 105-14.

18. American Society for Surgery of the Hand (ASSH). Clinical Assessment Committee Report. Rosemont: Churchill Livingstone; 1976.

19. Araújo PMP. Avaliação funcional. In: Freitas PP. Reabilitação da mão. $1^{a}$ ed. São Paulo: Atheneu; 2006. p. 35-54.

20. Fess EE. Documentation: Essential elements of an upper extremity assessment battery. In: Hunter JM, Schneider LH, Mackin EJ, Callahan AD, Skirven TM. Rehabilitation of the hand and upper extremity. $3^{\mathrm{a}}$ ed. St. Louis: Mosby; 2002. p. 263-83.
21. Oliveira LM, Araújo PMP. Medida de amplitude articular. In: Manual: Recomendações para avaliação do membro superior. $2^{a}$ ed. São Paulo: Sociedade Brasileira de Terapeutas da Mão; 2005. p. 42-54.

22. American Society for Surgery of the Hand (ASSH). The hand: examination and diagnosis. New York: Churchill Livingstone; 1983.

23. Delprat J, Mansat M, Erhler S. Evaluaciones articulares la muñeca y la mano. Encycl Med Chir. 1990;26:1-18.

24. Schall R. Estimation in generalized linear models with random effects. Biometrika. 1991;78:719-27.

25. Idler RS. Anatomy and biomechanics of the digital flexor tendons. Hand Clin. 1985;1:3-12.

26. Caetano BC. Anatomia functional da mão. In: Pardini AG. Traumatismos da mão. $3^{\text {a }}$ ed. Rio de Janeiro: Medsi; 2000. p. 7-78.

27. Kaplan EB, Hunter JM. Functional anatomy of the flexor tendon system: the muscle and tendon systems of the fingers. In: Hunter JM, Schneider LH, Mackin EJ. Tendon surgery in the hand. $1^{\mathrm{a}}$ ed. St. Louis: CV Mosby; 1987. p. 9-19.

28. Palastanga N, Field D, Soames R. Anatomia e movimento humano: estrutura e função. $3^{\text {a }}$ ed. São Paulo: Manole; 2000.

29. Ghez C. The control of movement. In: Kandel ER, Schwartz JH, Jessell TM. Principles of neural science. $3^{\mathrm{a}}$ ed. New York (NY): Elsevier Science; 1991. p. 533-47.

30. Gordon J, Ghez C. Muscle receptors and spinal reflexes: the stretch reflex. In: Kandel ER, Schwartz JH, Jessell TM. Principles of neural science. $3^{\text {a }}$ ed. New York (NY): Elsevier Science; 1991. p. 564-80.

31. Gordon J. Spinal mechanism of motor coordination. In: Kandel ER, Schwartz JH, Jessell TM. Principles of neural science. $3^{\text {a }}$ ed. New York (NY): Elsevier Science; 1991. p. 580-95.

32. Hogervorst T, Brand RA. Mechanoreceptors in joint function. J Bone Joint Surg Am. 1998;80:1365-78.

33. Wyke B. The neurology of joints. Ann R Coll Surg Engl. 1967;41:25-50.

34. Grigg P. Peripheral neural mechanisms in proprioception. J Sport Rehabil. 1994;3:2-17.

35. Baktir A, Türk CY, Kabak S, Sahin V, Kardas Y. Flexor tendon repair in zone II followed by early active mobilization. J Hand Surg. 1996;21B:624-8. 